\title{
DERMATITIS HERPETIFORMIS IN BULGARIA: REPORT OF 78 PATIENTS
}

\author{
M. Shahid, K. Drenovska, T. Velikova, S. Vassileva \\ Department of Dermatology, University Hospital “Alexandrovska”, Medical Faculty - Sofia, Bulgaria
}

\section{BACKGROUND \& OBJECTIVE}

Dermatitis herpetiformis (DH) is a chronic blistering dermatosis (AIBD) currently considered as the cutaneous manifestation of coeliac disease (CD). Both CD and DH are triggered by ingestion of wheat gluten and share a common immunogenetic background exhibited by a markedly increased inheritance of HLA-DQ2/DQ8 haplotypes. DH shows an uneven geographic and ethnic distribution, with higher incidence in Europe or populations with European descent. Most clinicoepidemiological features of DH, however, originate mainly from Northern Europe and UK but data are lacking from Eastern European countries. The aim of the present study was to analyze the clinical and immunopathological findings of DH patients in Bulgaria and to compare them with those reported from elsewhere.

\section{PATIENTS AND METHODS}

A retrospective analysis of the charts of all patients with DH diagnosed at the Department of Dermatology, University Hospital “Alexandrovska” between Jan 12005 - Dec 312016 was performed. During the 12-year period studied, a total of 78 patients were newly diagnosed with DH which represented $7.45 \%$ of 1047 cases of AIBD initially established at our immunopathology laboratory. The diagnosis was based on clinical, histologic, and direct immunofluorescence (IF) findings before initiation of gluten-free diet and treatment. Serum samples from 26 patients were analyzed for anti-endomysium antibodies (EMA) (indirect IF on monkey esophagus), antibodies against tissue transglutaminase (anti-tTG), deamidated gliadin peptides (anti-DGP), anti-actin (AAA) (enzyme-linked immunosorbent assay, ELISA), and anti-gliadin (AGA) (immunoblotting).

\section{RESULTS}

Patient characteristics.

Patients' demographic and clinical data are presented in Table 1. Most patients had DH in the fifth and sixth decades of life, with marked male predominance in the age groups 20-80 years (Fig. 1). All patients were Caucasians, predominantly of Bulgarian ethnic origin.

The most frequent clinical findings observed were a pruritic papulovesicular eruption symmetrically involving the extensor surfaces of the extremities (Fig. 2), the back, buttocks, groin, but also the face and scalp (Fig. 3).

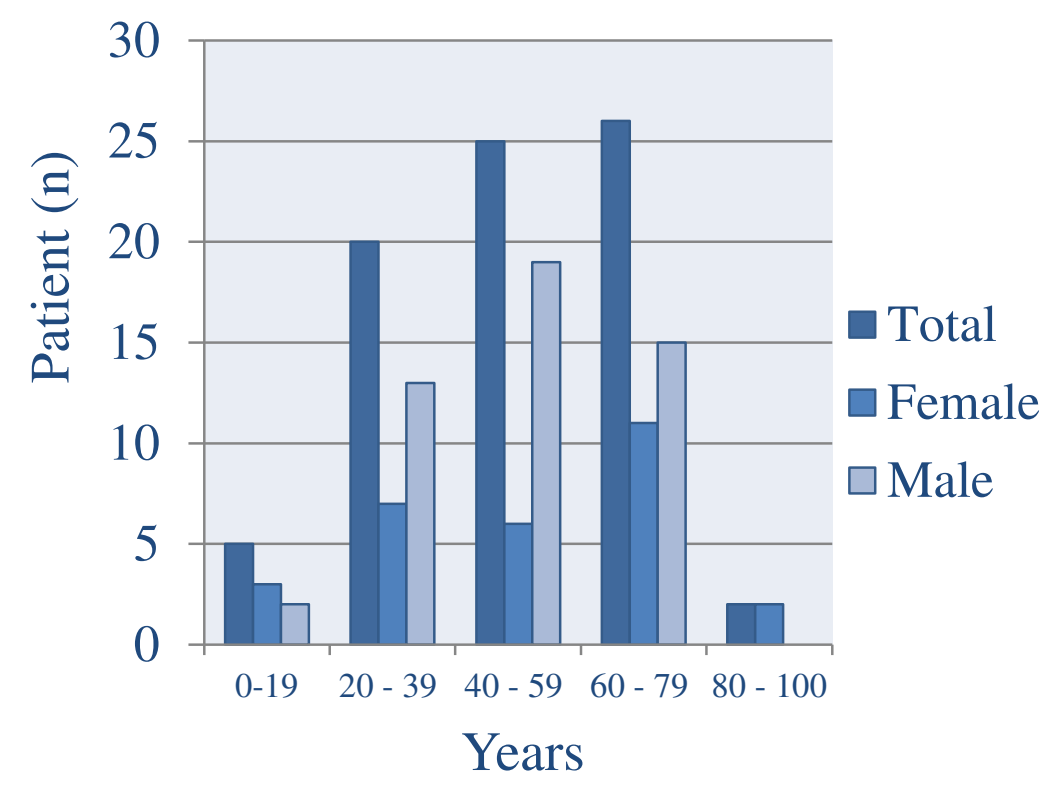

Fig. 1. Age and sex distribution

Table 2. Direct immunofluorescence findings

\begin{tabular}{|l|l|l|}
\hline Type of deposits & Granular & Fibrillar \\
\hline IgA & 77 & 1 \\
\hline Other deposits & 55 & 0 \\
\hline IgG & 12 & 0 \\
\hline IgM & 19 & 0 \\
\hline C3 & 47 & 0 \\
\hline Deposits at DEJ & 6 & 1 \\
\hline $\begin{array}{l}\text { Deposits at tips of } \\
\text { dermal papillae }\end{array}$ & 29 & 0 \\
\hline Deposits at both & 42 & 0 \\
\hline Deposits in vessels & 5 & 0 \\
\hline
\end{tabular}
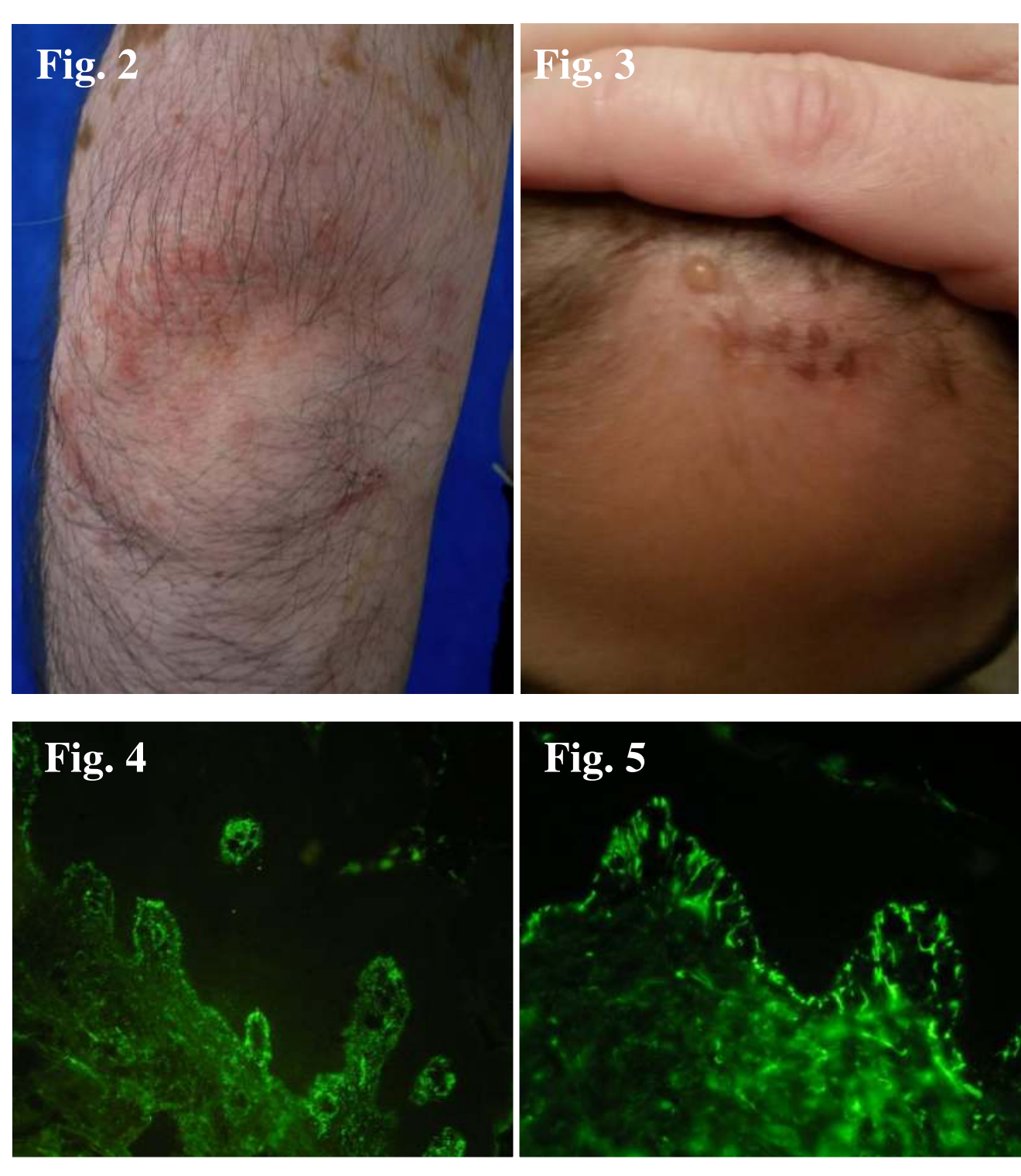

Direct immunofluorescence results

Direct immunofluorescence (DIF) microscopy revealed granular deposits of IgA at the papillary tips (Fig. 4) andor along the dermal-epidermal junction in $98.75 \%$ (77/78) of cases, and fibrillar IgA (Fig. 5) in 1 patient (a 13-year-old boy presented on Fig. 3). The distribution and types of immunoreactants are detailed in Table 2.

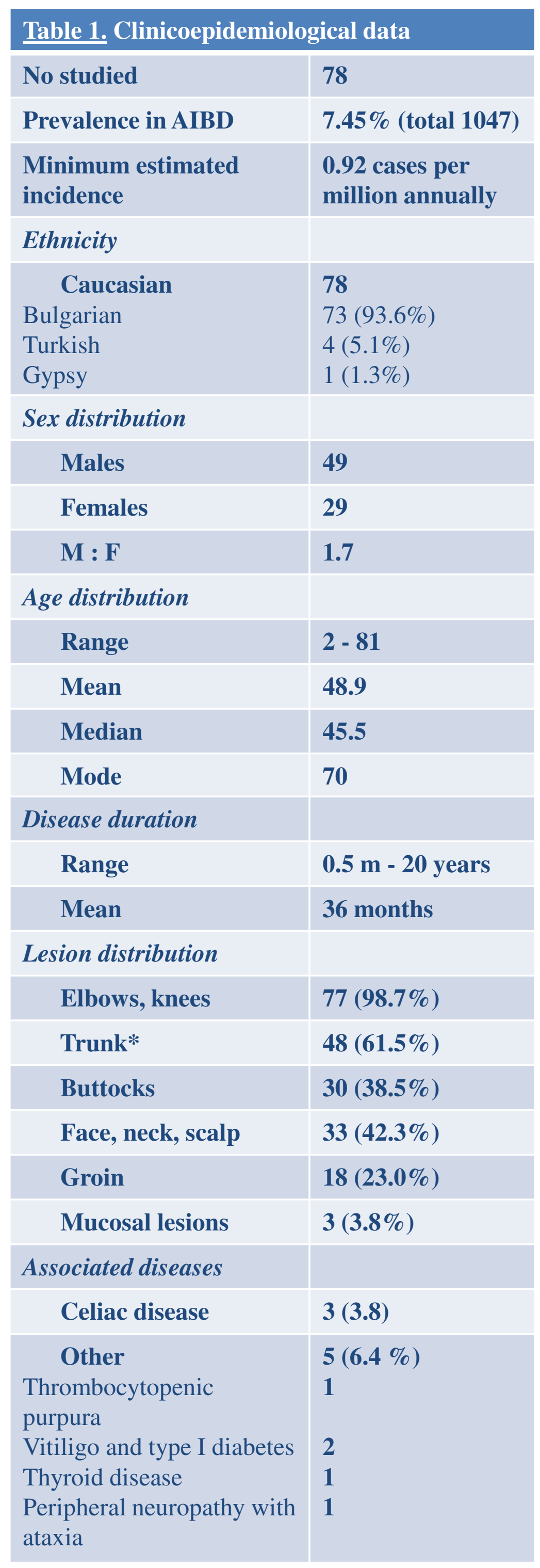

AIBDs, autoimmune blistering diseases * Excluding buttocks

\section{Immune serology}

IgA-EMA were positive in 52\% of sera. Anti-tTG were found in 11 (42.3\%) DH patients. Half of the patients had increased values of AGA in their sera, 12 (46.4\%) were positive for anti-DGP, whereas only $9(34.7 \%)$ were positive for AAA.

\section{CONCLUSION}

Our series of DH patients, which is the first one reported from Bulgaria, confirmed the data from the literature, namely the prevalence of granular type of immune deposits in Caucasian patients. Our results also showed that immunoserologic testing for a panel of autoantibodies associated with CD may be successfully employed for diagnosing $\mathrm{DH}$ as a non-invasive addition to immunopathologic diagnostic criteria.

\section{REFERENCES}

1. Salmi TT, et al. Prevalence and incidence of dermatitis herpetiformis: a 40-year prospective study from Finland. Br J Dermatol. 2011;165:354-9.

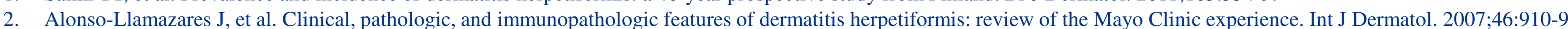

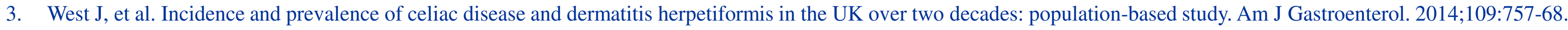

